\title{
Differential expression of NIMA-related kinase 2 in triple negative breast cancer.
}

Shahan Mamoor, MS ${ }^{1}$

$3 \quad$ shahanmamoor@gmail.com

East Islip, NY 11730

Women diagnosed with triple negative breast cancer can benefit neither from endocrine therapy nor from HER2-targeted therapies ${ }^{1}$. We mined published microarray datasets ${ }^{2,3}$ to determine in an unbiased fashion and at the systems level genes most differentially expressed in the primary tumors of patients with breast cancer. We report here significant differential expression of the gene encoding NIMA-related kinase 2, NEK2, when comparing the tumor cells of patients with triple negative breast cancer to normal mammary ductal cells ${ }^{2}$. NEK2 was also differentially expressed in bulk tumor in human breast cancer ${ }^{3}$. NEK2 mRNA was present at significantly increased quantities in TNBC tumor cells relative to normal mammary ductal cells. Analysis of human survival data revealed that expression of NEK2 in primary tumors of the breast was correlated with distant metastasis-free survival in patients with luminal A type cancer, while within triple negative breast cancer, primary tumor expression of NEK2 was correlated with overall survival in patients with luminal androgen receptor and mesenchymal subtype disease. NEK2 may be of relevance to initiation, maintenance or progression of triple negative breast cancers.

Keywords: breast cancer, NEK2, NIMA-related kinase 2, systems biology of breast cancer, targeted therapeutics in breast cancer. 
Patients with hormone receptor positive disease can benefit from endocrine therapy ${ }^{4}$ : aromatase inhibition such as that provided by exemestane or letrozole, or estrogen receptor antagonism such as that provided by tamoxifen or fulvestrant. Patients whose tumors overexpress the human epidermal growth factor 2 (HER2) can be treated with HER2-targeted therapies: HER2 inhibitors, immunoglobulin ${ }^{5}$ - or small molecule-based ${ }^{6}$. As triple negative breast cancer (TNBC) is defined by its lack of HER2 expression as well as by its lack of expression of the hormone receptor estrogen, patients diagnosed with triple negative breast cancer will not benefit from either of these treatment strategies.

Understanding the transcriptional composition of triple negative breast cancers in humans can facilitate development of novel therapeutics and contribute to efforts to prevent progression to metastatic stages. We mined published microarray data ${ }^{2,3}$ to understand at the transcriptome level and in an unbiased fashion genes most differentially expressed in the tumor cells of patients with triple negative breast cancer.

\section{Methods}

We utilized datasets GSE38959 ${ }^{2}$ and GSE109169³ for this differential gene expression analysis of female breast cancer in conjunction with GEO2R. GSE38959 was generated using Agilent-014850 Whole Human Genome Microarray 4x44K G4112F technology with $n=13$ samples of normal mammary gland ductal cells and $n=30$ samples of tumor cells from patients with triple negative breast cancer; analysis was performed using platform GPL4133. GSE109169 was generated using Affymetrix Human Exon 1.0 ST Array technology with $n=25$ normal breast tissue and $n=25$ tumors of the breast; analysis was performed using platform GPL5175. The tissues from this dataset are paired tissues ( 25 tumors matching 25 normal breast tissues from 25 patients). The Benjamini and Hochberg method of $p$-value adjustment was used for ranking of differential expression but raw $p$-values were used to assess statistical significance of global differential expression. Log-transformation of data was auto-detected, and the NCBI-generated category of platform annotation was used. A statistical test was performed to evaluate whether NEK2 expression was significantly different between primary breast tumors and breast tissue using a two-tailed t-test.

For Kaplan-Meier survival analysis, we used the Kaplan-Meier plotter tool ${ }^{7}$ for correlation of NEK 2 mRNA expression levels with distant metastasis-free survival (DMFS) in $n=630$ patients with basal subtype cancer, $n=998$ patients with luminal A subtype cancer, $n=673$ patients with luminal B subtype cancer, $n=401$ patients with HER $2+$ cancer, and $n=999$ patients with normal-like subtype cancer. In patients with triple negative breast cancer ${ }^{7}$, we analyzed overall survival (OS) for $n=103$ patients with basal-like 1 subtype TNBC, $n=58$ patients with basal-like 2 subtype TNBC, $n=149$ patients with immunomodulatory subtype TNBC, $n=116$ patients with luminal androgen receptor subtype TNBC, $n=114$ patients with mesenchymal subtype TNBC, and $n=39$ patients with mesenchymal stem-like subtype TNBC.

\section{Results}

We performed discovery of genes associated with triple negative breast cancer in humans by mining two independently published microarray datasets ${ }^{2,3}$.

\section{NEK2 is differentially expressed in primary tumors and tumor cells of the breast.}

Comparison of the tumor cells of patients with triple negative breast cancer to normal mammary ductal cells ${ }^{2}$ revealed NIMA-related kinase 2, encoded by NEK2, as among the genes whose expression was most significantly different in human TNBC as compared to the normal breast (Chart 1). When sorting each of the genes expressed in the tumor cells of patients with triple negative breast cancer based on significance of difference as compared to normal mammary ductal cells, NEK2 ranked 3 out of 45015 total transcripts, equating to $99.99 \%$ differential expression (Chart 1). Differential expression of NEK2 in the tumor cells of patients with triple negative breast cancer was statistically significant (Chart 1; 
$p=3.83 \mathrm{E}-16)$.

Analysis of a second microarray datase ${ }^{3}$, here comparing 25 normal breast tissues to 25 tumors of the breast revealed that NEK2 was also among the genes most differentially expressed in human breast cancer, here in the tumors of women diagnosed with early-onset breast cancer (Chart 2). When sorting each of the genes expressed in tumors of the breast based on significance of difference as compared to normal breast tissue, NEK2 ranked 567 out of 19076 total transcripts, equating to $97.0 \%$ differential expression (Chart 2). Differential expression of NEK2 in female breast cancer was statistically significant (Chart 2; $p=2.18 \mathrm{E}-10)$.

These data indicated that differential expression of NEK2 was not an artifact of a single microarray dataset, was not an artifact of FACS isolation, and suggested that perturbed expression of NEK2 was likely a transcriptional feature of triple negative breast cancers and early-onset human breast cancers.

NEK2 is expressed at significantly higher levels in triple negative tumor cells as compared to normal cells of the breast.

We obtained exact mRNA expression levels for NEK2 - from normal mammary ductal cells of the breast and from tumor cells of patients with triple negative breast cancer - to understand the magnitude and direction of NEK2 expression change in TNBC. NEK2 mRNA transcript was present at higher levels in triple negative tumor cells of the breast as compared to normal mammary ductal cells (Figure 1). Increased expression of NEK2 in primary tumor-isolated triple negative breast cancer cells was statistically significant (Figure 1: $p<0.0001$ ). NEK2 was expressed at $19.7 \pm 48.6$ arbitrary units (A.U.) in normal mammary ductal cells, while it was expressed at $201.8 \pm 104.3$ A.U. in tumor cells of patients with triple negative breast cancer. We calculated a mean fold change of 10.2 when comparing triple negative breast cancer cells to normal mammary ductal cells.

\section{NEK2 expression correlates with survival outcomes in luminal A human breast cancer.}

We performed Kaplan-Meier survival analysis to evaluate potential relationships between primary tumor NEK2 mRNA expression levels and survival outcomes in patients with breast cancer, when analyzing outcomes based on the molecular subtype of the patient (PAM50 classification): luminal A, luminal B, HER2+, normal-like, and basal-like. We observed a correlation between NEK2 expression and distant metastasis-free survival (DMFS) in patients with luminal A subtype breast cancer which trended towards statistical significance (Figure 2; $\log$ rank $p$-value: 0.073 for distant metastasis-free survival, hazard ratio: 1.35 (0.97-1.86) (Fig. 2)).

NEK2 mRNA levels were a negative prognostic indicator in luminal A breast cancer patients. Median DMFS was 149 months for luminal A patients with low tumor expression of NEK2 while median DMFS was 142.39 months for luminal A patients with high tumor expression of NEK2 (Chart 3).

NEK2 primary tumor expression was not correlated with distant metastasis-free survival in basal subtype (Figure 2; log rank $p$-value: 0.75 for DMFS, hazard ratio: 1.05 (0.78-1.41) (Fig. 2), luminal B subtype (Figure 2; log rank $p$-value: 0.42 for DMFS, hazard ratio: 1.13 (0.84-1.51) (Fig. 2)), in HER2+ breast cancer (Figure 2; log rank $p$-value: 0.46 for DMFS, hazard ratio: 0.88 (0.62-1.24) (Fig. 2)), or in the normal-like subtype (Figure 2; log rank $p$-value: 0.84 for DMFS, hazard ratio: 0.91 (0.34-2.4) (Fig. 2)).

\section{Influence of primary tumor NEK2 expression on survival outcomes based on TNBC molecular subtype.}

We also performed Kaplan-Meier survival analysis to examine relationships between NEK2 
outcomes based on the TNBC molecular subtype of the patient: basal-like 1, basal-like 2, immunomodulatory, mesenchymal, mesenchymal stem-like, and luminal androgen receptor. We observed a statistically significant correlation between NEK2 expression and overall survival (OS) in TNBC patients with luminal androgen receptor subtype disease (Figure 3; log rank $p$-value: 0.015 for overall survival, hazard ratio: 0.47 (0.25-0.88) (Fig. 3)). NEK2 mRNA levels were a positive prognostic indicator in patients with luminal androgen receptor TNBC. Median OS was 36.03 months for luminal androgen receptor TNBC patients with low tumor expression of NEK2 while median OS was 56.64 months for luminal androgen receptor TNBC patients with high tumor expression of NEK2 (Chart 4).

We also observed a correlation between NEK2 expression and overall survival (OS) in TNBC patients with mesenchymal subtype disease which trended towards statistical significance (Figure 3; log rank $p$-value: 0.079 for overall survival, hazard ratio: 0.55 (0.28-1.08) (Fig. 3)). NEK2 mRNA levels were a positive prognostic indicator in patients with mesenchymal TNBC. Median OS was 30.13 months for mesenchymal TNBC patients with low tumor expression of NEK2 while median OS was 59.88 months for mesenchymal TNBC patients with high tumor expression of NEK2 (Chart 4).

NEK2 primary tumor expression was not correlated with overall survival in the basal-like 1 subtype (Figure 3; log rank $p$-value: 0.56 for OS, hazard ratio: 0.79 (0.36-1.73) (Fig. 3)), basal-like 2 subtype (Figure 3; log rank $p$-value: 0.7 for OS, hazard ratio: 0.82 (0.31-2.22) (Fig. 3), immunomodulatory subtype (Figure 3; $\log$ rank $p$-value: 0.94 for OS, hazard ratio: 0.97 (0.44-2.13) (Fig. $3)$ ), or in the mesenchymal stem-like subtype (Figure 3; log rank $p$-value: 0.17 for OS, hazard ratio: 0.47 (0.16-1.4) (Fig. 3)).

\section{Discussion}

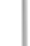

We performed comparative transcriptome analysis using two independently published microarray datasets $^{2,3}$ to define and catalog transcriptional differences in human triple negative breast cancer. We provided evidence here that NIMA-related kinase 2, encoded by the NEK2 gene, is a defining transcriptional feature of triple negative breast cancer. NEK2 was also differentially expressed in the bulk tumors of patients with early-onset breast cancer. Expression of NEK2 was correlated with distant metastasis-free survival in patients with luminal A subtype breast cancer, and within triple negative breast cancer patients, primary tumor expression of NEK 2 was correlated with overall survival in patients with luminal androgen receptor subtype and mesenchymal subtype disease. NIMA-related kinase 2 and the molecular processes to which it pertains to may be relevant to the initiation or progression of triple negative breast cancer in humans. 


\section{References}

1. Foulkes, W.D., Smith, I.E. and Reis-Filho, J.S., 2010. Triple-negative breast cancer. New England journal of medicine, 363(20), pp.1938-1948.

2. Komatsu, M., Yoshimaru, T., Matsuo, T., Kiyotani, K., Miyoshi, Y., Tanahashi, T., Rokutan, K., Yamaguchi, R., Saito, A., Imoto, S. and Miyano, S., 2013. Molecular features of triple negative breast cancer cells by genome-wide gene expression profiling analysis. International journal of oncology, 42(2), pp.478-506.

3. Chang, J.W., Kuo, W.H., Lin, C.M., Chen, W.L., Chan, S.H., Chiu, M.F., Chang, I.S., Jiang, S.S., Tsai, F.Y., Chen, C.H. and Huang, P.H., 2018. Wild-type p53 upregulates an early onset breast cancer-associated gene GAS7 to suppress metastasis via GAS7-CYFIP1- mediated signaling pathway. Oncogene, 37(30), pp.4137-4150.

4. Spring, L.M., Gupta, A., Reynolds, K.L., Gadd, M.A., Ellisen, L.W., Isakoff, S.J., Moy, B. and Bardia, A., 2016. Neoadjuvant endocrine therapy for estrogen receptor-positive breast cancer: a systematic review and meta-analysis. JAMA oncology, 2(11), pp.1477-1486.

5. Slamon, D., Eiermann, W., Robert, N., Pienkowski, T., Martin, M., Press, M., Mackey, J., Glaspy, J., Chan, A., Pawlicki, M. and Pinter, T., 2011. Adjuvant trastuzumab in HER2-positive breast cancer. New England journal of medicine, 365(14), pp.1273-1283.

6. Park, J.W., Liu, M.C., Yee, D., Yau, C., van’t Veer, L.J., Symmans, W.F., Paoloni, M., Perlmutter, J., Hylton, N.M., Hogarth, M. and DeMichele, A., 2016. Adaptive randomization of neratinib in early breast cancer. New England Journal of Medicine, 375(1), pp.11-22.

7. Györffy, B., Lanczky, A., Eklund, A.C., Denkert, C., Budczies, J., Li, Q. and Szallasi, Z., 2010. An online survival analysis tool to rapidly assess the effect of 22,277 genes on breast cancer prognosis using microarray data of 1,809 patients. Breast cancer research and treatment, 123(3), pp.725-731. 


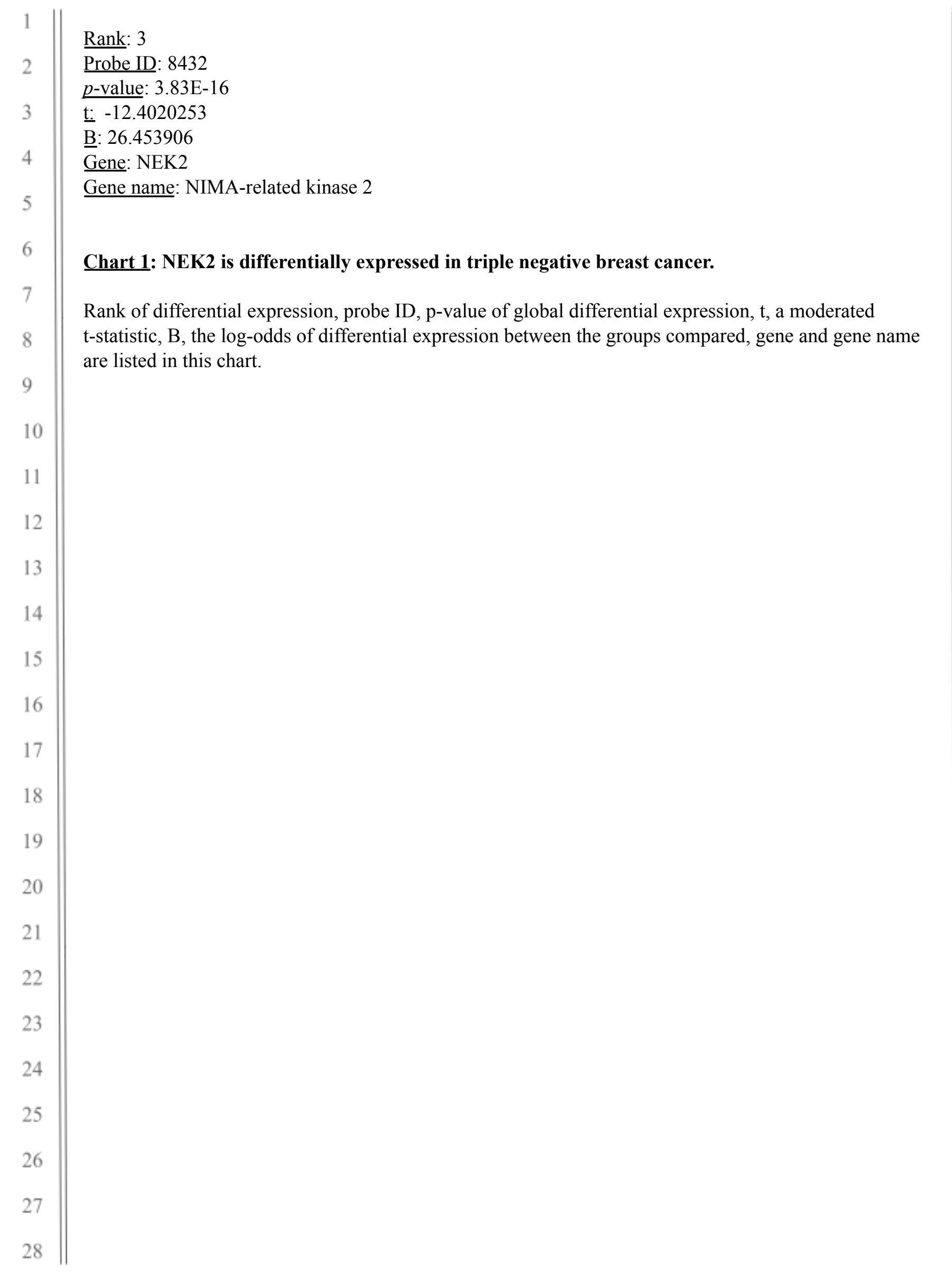




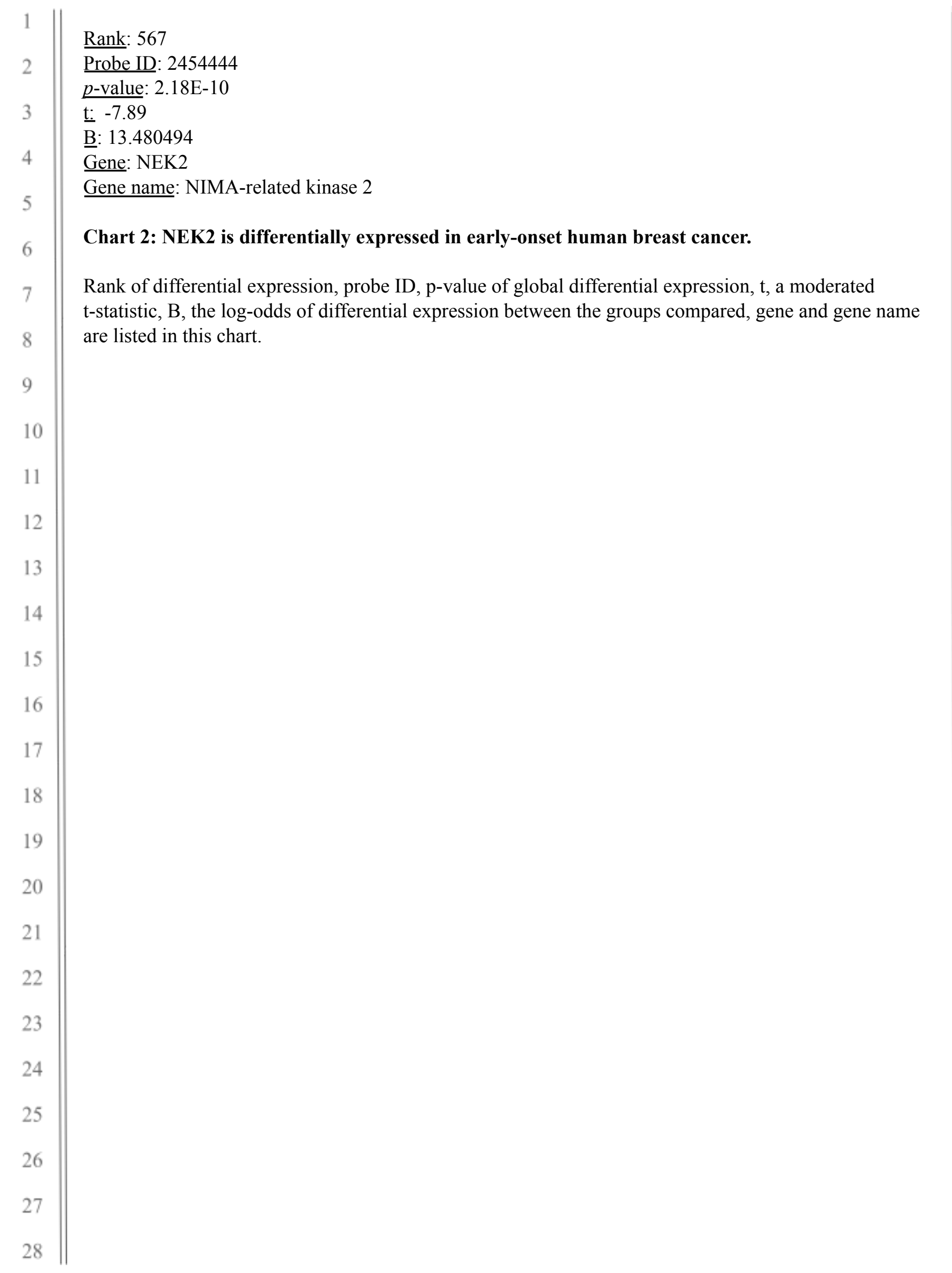




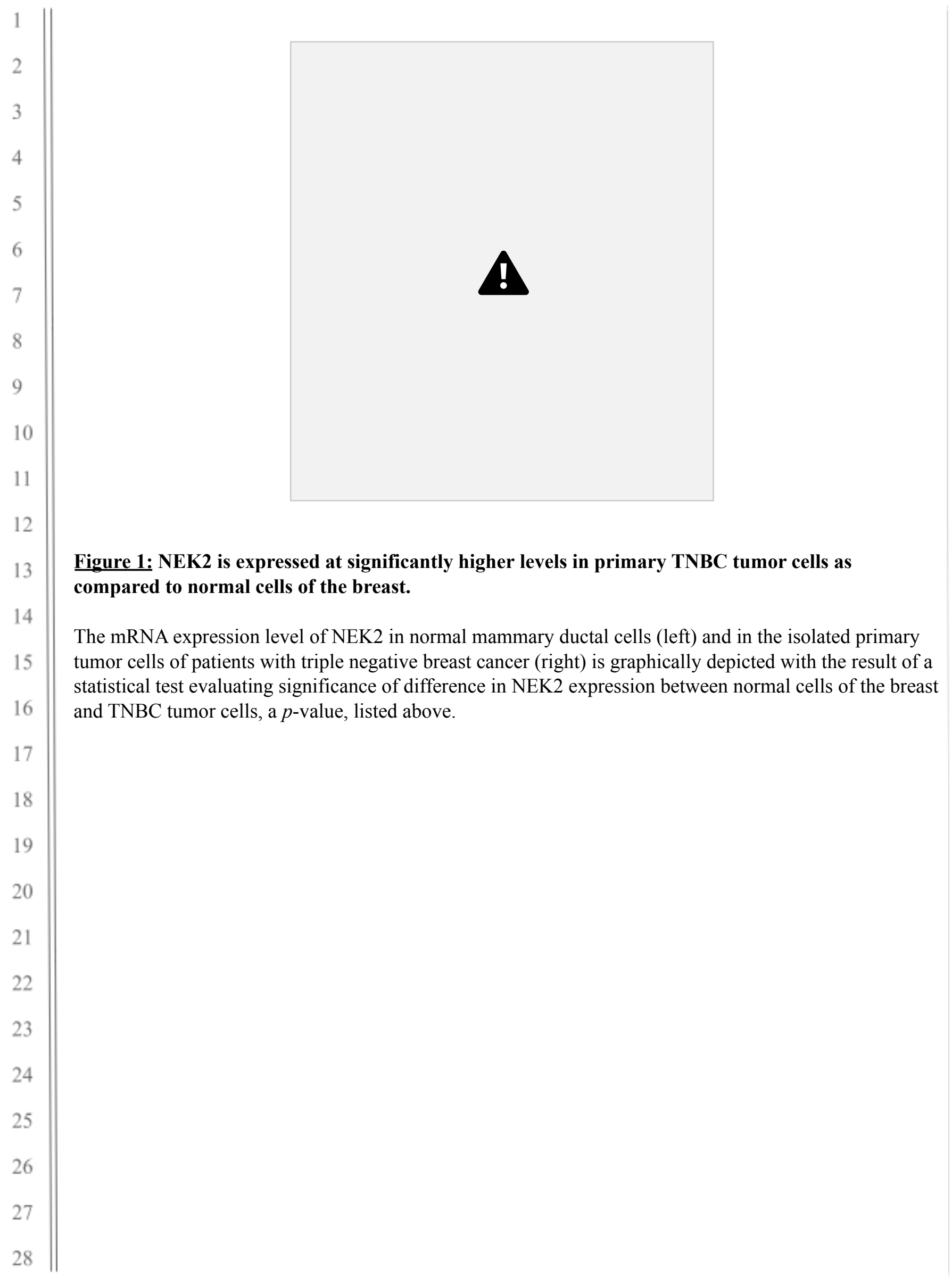



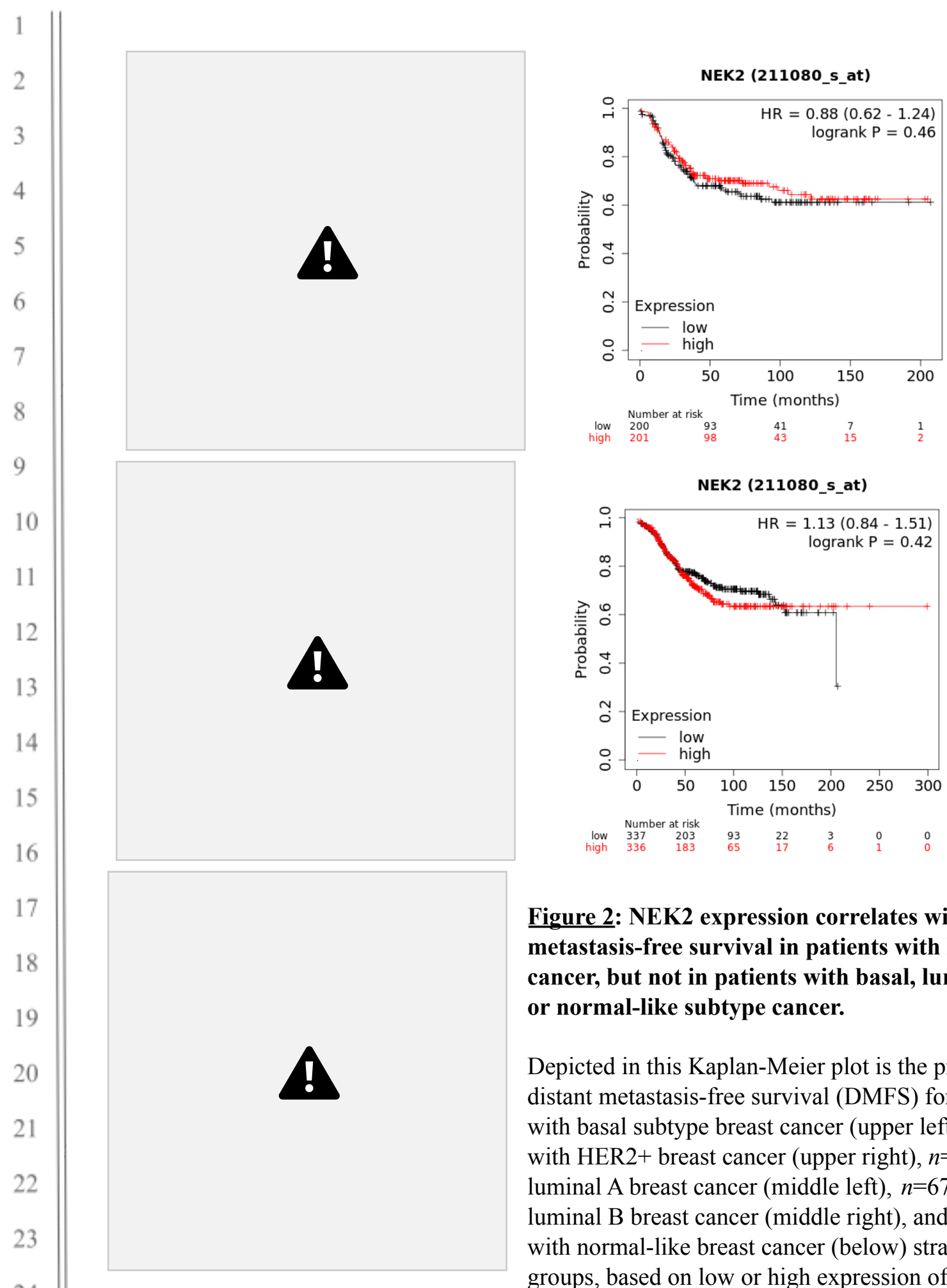

Figure 2: NEK2 expression correlates with distant metastasis-free survival in patients with luminal $A$ breast cancer, but not in patients with basal, luminal B, HER2+, or normal-like subtype cancer.

Depicted in this Kaplan-Meier plot is the probability of distant metastasis-free survival (DMFS) for $n=630$ patients with basal subtype breast cancer (upper left), $n=401$ patients with HER2 + breast cancer (upper right), $n=998$ patients with luminal A breast cancer (middle left), $n=673$ patients with luminal B breast cancer (middle right), and $n=999$ patients with normal-like breast cancer (below) stratified into two groups, based on low or high expression of NEK2 in patient primary tumors. The log rank $p$-value denoting statistical significance of difference in distant metastasis-free survival when comparing the two groups, as well as hazard ratio for this comparison is listed above. Listed below is the number of patients at risk (number of patients alive) per interval, after stratification based on NEK2 expression; in the first interval, number at risk is number of patients alive; in each subsequent interval, number at risk is the number at risk less those who have expired or are censored. 







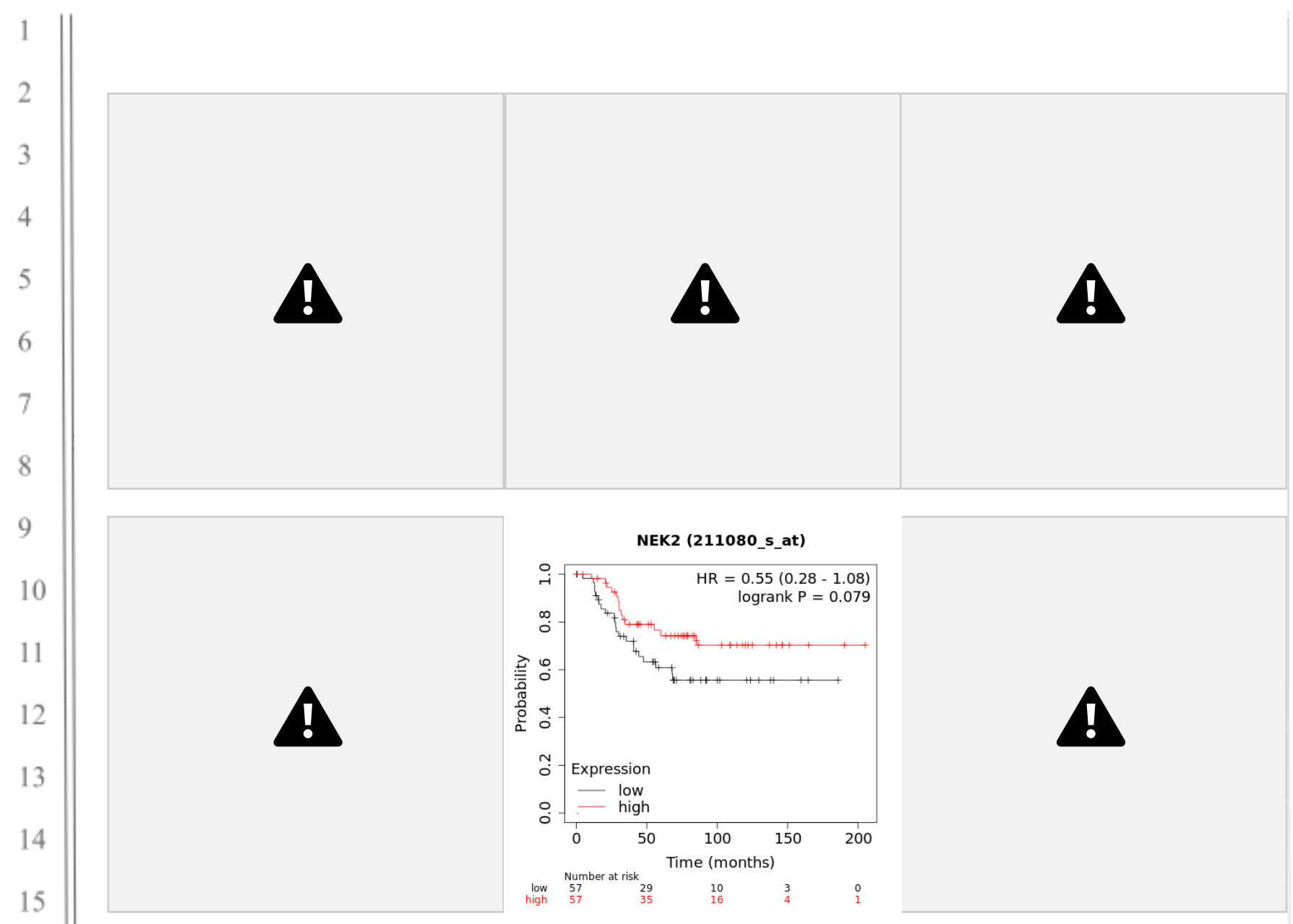

Figure 3: NEK2 expression correlates with overall survival in triple negative breast cancer patients whose tumors are of the luminal androgen receptor and mesenchymal molecular subtypes.

Depicted in this Kaplan-Meier plot is the probability of overall survival (OS) for $n=103$ patients with basal-like 1 subtype TNBC (upper left), $n=58$ patients with basal-like 2 subtype TNBC (upper middle), $n=149$ patients with immunomodulatory subtype TNBC (upper right), $n=116$ patients with luminal androgen receptor subtype TNBC (lower left), $n=114$ patients with mesenchymal subtype TNBC (lower middle), and $n=39$ patients with mesenchymal stem-like subtype TNBC (lower right), stratified into two groups, based on low or high expression of NEK2 in the primary tumors of patients with TNBC. The log rank p-value denoting statistical significance of difference in overall survival when comparing the two groups, as well as hazard ratio for this comparison is listed above. Listed below is the number of patients at risk (number of patients alive) per interval, after stratification based on NEK2 expression; in the first interval, number at risk is number of patients alive; in each subsequent interval, number at risk is the number at risk less those who have expired or are censored. 


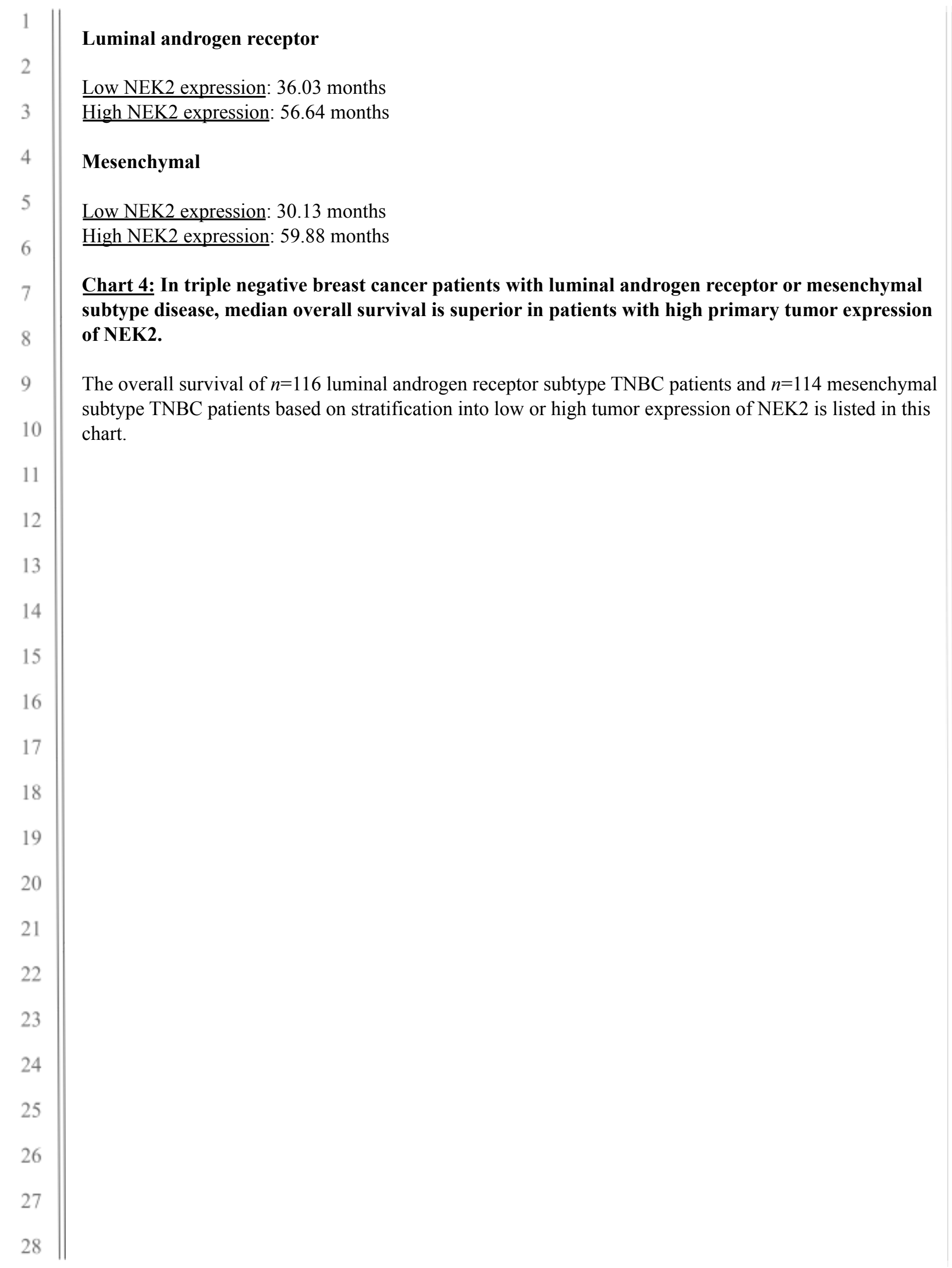

